\title{
Evidence of structural modifications in the region around the broad dielectric maxima in the $30 \%$ Sn-doped barium titanate relaxor
}

\author{
Akash Surampalli, ${ }^{1}$ Irene Schiesaro, ${ }^{2}$ Pietro Corsi, ${ }^{2}$ Carlo Meneghini,,${ }^{2, *}$ V. G. Sathe, ${ }^{1}$ Archna Sagdeo, ${ }^{3,4}$ A. K. Sinha, ${ }^{3,4}$ \\ Giuliana Aquilanti, ${ }^{5}$ Edmund Welter, ${ }^{6}$ and V. Raghavendra Reddy ${ }^{1, \dagger}$ \\ ${ }^{1}$ UGC-DAE Consortium for Scientific Research, University Campus, Khandwa Road, Indore 452001, India \\ ${ }^{2}$ Dipartimento di Scienze, Universita di Roma Tre, I-00146 Roma, Italy \\ ${ }^{3}$ Synchrotron Utilization Section, RRCAT, Indore India \\ ${ }^{4}$ Homi Bhabha National Institute, Training School Complex, Anushakti Nagar, Mumbai 400094, India \\ ${ }^{5}$ Sincrotrone Trieste, SS 14, Km 163.5, I-34012 Basovizza, Trieste, Italy \\ ${ }^{6}$ Deutsches Elektronen-Synchrotron - A Research Centre of the Helmholtz Association, Hamburg 22607, Germany
}

(Received 27 June 2019; revised manuscript received 24 September 2019; published 18 October 2019)

\begin{abstract}
The structural and dielectric characterization of $30 \%$ Sn-doped $\mathrm{BaTiO}_{3}\left(\mathrm{BaSn}_{0.3} \mathrm{Ti}_{0.7} \mathrm{O}_{3}\right)$ as a function of temperature is carried out combining, complementary probes to reveal structural modifications associated to the relaxor behavior. Dielectric data confirms the existence of relaxor-type behavior in $\mathrm{BaSn}_{0.3} \mathrm{Ti}_{0.7} \mathrm{O}_{3}$. The local and average structural changes as a function of temperature have been investigated in the region of broad changes of dielectric response. Local probes, such as x-ray absorption fine structure spectroscopy and Mössbauer spectroscopy reveal a special trend of the lattice related to the diffuse dielectric phase transition as observed from dielectric data. The analysis of Raman spectra as a function of temperature reveals a peculiar behavior in the temperature region corresponding to the dielectric transition. The analysis of $\mathrm{x}$-ray diffraction patterns points out a pseudocubic structure throughout the temperature range while the volume exhibits a negative thermal expansion in the region of broad dielectric maximum. Our results suggest structural modifications occurring in the system throughout a wide temperature range, interestingly, associated with changes in macroscopic properties of relaxors such as dispersion in dielectric constants and raising of dipole moments.
\end{abstract}

DOI: 10.1103/PhysRevB.100.134104

\section{INTRODUCTION}

Lead-free $\mathrm{BaTiO}_{3}$ (BTO)-based ferroelectric (FE) ceramics have been explored the most due to their potential applications in the fields of spacecrafts, renewable energy, etc. Also, they have drawn a lot of attention in fundamental research due to their existence in different phases across a wide range of temperatures. Researchers have been studying the effects on phase transitions and dielectric permittivity by manipulating the $\mathrm{Ba}$ and $\mathrm{Ti}$ sites with doping and found enhanced electrocaloric, piezoelectric properties [1,2]. A specific class of such FE materials, relaxors, have gained a lot of attention due to their high dielectric permittivity and a broad frequency dispersive permittivity peak versus temperature [3]. It was suggested that the existence of polar nanoregions (PNRs) are responsible for frequency dispersion of dielectric constants [3]. The indirect existence of PNRs is confirmed through diffuse scattering and transmission electron microscopy experiments in both BTO and Pb-based FE materials $[4,5]$. Since then, theories to understand the origin and evolution of PNRs as a function of temperature have been developed but are still in debate [6-12]. It was suggested that PNRs of high frequency polarization fluctuations are generated below $T_{d}$ (Burns temperature) [3] and, on further lowering temperature,

\footnotetext{
*carlo.meneghini@uniroma3.it; cmeneghi@gmail.com

†varimalla@yahoo.com; vrreddy@csr.res.in
}

the relaxation time increases and diverges, causing freezing of PNRs corresponding to a freezing temperature $T_{f}$. It was also suggested there exists an intermediate temperature $T^{*}$ associated with an increase in dipole moments, dispersion in dielectric constants, and also characterized by changing local structural dynamics around that temperature in $\mathrm{Pb}$-based $\mathrm{FE}$ materials [13-15]. One of the earliest models about the formation of PNRs and their temperature dynamics in $A(B \bar{B}) O_{3}$ perovskite invokes the role of random bond fields (isovalent $B$ site substitution with $B$ ) and random electric fields (heterovalent $B$ site substitution with $B$ ) $[11,16]$. This random field model, which is a phenomenological model, explains the anomaly observed in specific heat around Burns temperature [3,17], the dielectric dispersion, the electric-field-induced susceptibility change [18] and the characteristic temperatures describing PNR dynamics $[13,18]$ in Pb-based FE materials where there is a nominal charge disorder. However, the absence of specific heat anomaly around Burns temperature [19] in the $\mathrm{Pb}$-free relaxor $\mathrm{BaTi}_{0.65} \mathrm{Zr}_{0.35} \mathrm{O}_{3}$ raises the question about the role of random fields as the driving force responsible for the nucleation of PNRs and their dynamics. A recent discovery of soft mode in $\mathrm{PbMg}_{1 / 3} \mathrm{Nb}_{2 / 3} \mathrm{O}_{3}$ (PMN) [14] and recent atomistic and first-principles calculations in BTO-based relaxors [7,12] revealed that local structural changes and the displacive nature of FE play an important role in the formation of PNRs and their dynamics. The role of coupling between $B$ and $B$ in $A(B \bar{B}) O_{3}$ perovskite and the temperature evolution of their correlation parameter is also widely discussed in the context 
of relaxor properties $[7,8,20]$. In view of these recent theoretical predictions and experimental evidence, we attempted to deeper investigate the role of the local atomic structure and lattice dynamics of the relaxor compound $\mathrm{BaTi}_{0.7} \mathrm{Sn}_{0.3} \mathrm{O}_{3}$, combining complementary probes such as $\mathrm{x}$-ray diffraction (XRD), Raman spectroscopy, x-ray absorption spectroscopy (XAS) and ${ }^{119} \mathrm{Sn}$ Mössbauer spectroscopy, focusing on the region around dielectric maxima. The measurements provide a deeper experimental insight into the local atomic structure associated to the PNR dynamics in the temperature region of the dielectric transition of BTO-based relaxors.

\section{EXPERIMENTAL DETAILS}

Polycrystalline $30 \%$ Sn-doped $\mathrm{BaTiO}_{3}$ is prepared with a conventional solid-sate sintering method starting from high purity $(\geqslant 99.9 \%)$ oxide and carbonate precursors. Dielectric measurements were performed using a precision LCR meter E4980 and an in-house developed sample holder with $1 \mathrm{~V}$ $\mathrm{AC}$ signal at different frequencies in the temperature range 40-300 K, while cooling the sample. Temperature-dependent P-E hysteresis loops were measured using a Radiant Precision Premier II FE loop tracer using liquid nitrogen as cryogen in the temperature range of $80-350 \mathrm{~K}$ and placing the sample in a Delta- 9023 chamber. Temperature-dependent XRD studies were carried out at the BL-12 beamline of the Indus-2 synchrotron radiation facility (Indore, India) in the temperature range 50-300 K. Temperature-dependent Raman spectra were recorded with LabRam-HR800 in backscattering geometry equipped with the diode laser $(\lambda=473 \mathrm{~nm})$ as the excitation source in the temperature range 10-300 K, using a liquid helium flow cryostat. Temperature-dependent XAS data were collected in transmission geometry at ELETTRA (Trieste, Italy) 11.1R beamline [21] (Ti $K$ edge) and PETRAIII, DESY (Hamburg, Germany) P65 beamline (Sn $K$ edge) in the temperature range $20-300 \mathrm{~K}$ and $10-300 \mathrm{~K}$ respectively. Temperature-dependent ${ }^{119} \mathrm{Sn}$ Mössbauer measurements are carried out in transmission mode using a standard PC-based Mössbauer spectrometer equipped with a WisEl velocity drive in constant acceleration mode and placing the sample in a Janis CCR system. The spectrometer is calibrated with natural iron and the reported isomer shift values are with respect to $\mathrm{SnO}_{2}$.

\section{RESULTS AND DISCUSSIONS}

\section{A. Dielectric characterization}

The dielectric studies of BTO with various doping elements are very well reported and our experimental findings, as shown in Fig. 1, very well match with the literature [22,23]. The dielectric maximum temperature $\left(T_{m}\right)$ is found to shift toward higher temperature with increasing frequency, which is a characteristic of relaxor FEs [3]. According to the literature [13], the $T^{*} \sim 180 \mathrm{~K}$ temperature is assigned, signaling the onset of dispersion in $\epsilon^{\prime}$ and increase in $\epsilon^{\prime \prime}$. The dispersion in dielectric constant associated with raising dielectric losses below $T^{*}$ directly points out the growth of PNRs with finite relaxation times. The trend of $T_{m}$ as a function of frequencies is fitted, as shown in the inset of Fig. 1 using the Vogel-Fulcher

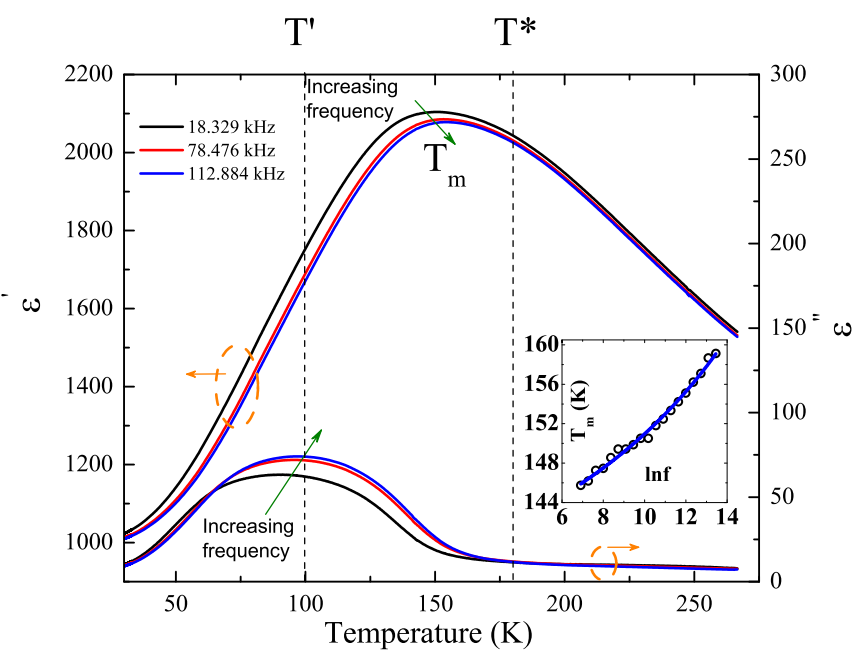

FIG. 1. Temperature-dependent dielectric constant and $\varepsilon^{\prime \prime}$ of $\mathrm{BaTi}_{0.7} \mathrm{Sn}_{0.3} \mathrm{O}_{3}$ at different frequencies. Inset shows the VogelFulcher fit to the variation of $T_{m}$ as a function of frequency as discussed in the text.

(VF) formalism defined as

$$
\tau=\tau_{o} \exp \frac{E}{k\left(T_{m}-T_{f}\right)},
$$

where $\tau$, relaxation time, is taken as inverse of probing frequencies. The freezing temperature $\left(T_{f}\right)$, being the temperature below which PNRs become static (freeze) and relaxation time diverges, is obtained from the VF fit as shown in the inset of Fig. 1, resulting around $120 \pm 5 \mathrm{~K}$. However, it is clear from the dielectric spectra (Fig. 1) that there is still significant dispersion in real and imaginary parts of dielectric constants below $T_{f}$ and the $\epsilon^{\prime \prime}$ reaches a broad maximum around $100 \mathrm{~K}$ (low frequency) and significantly decreases only around $\sim 70 \mathrm{~K}$ associated with the vanishing frequency dispersion of $\epsilon^{\prime \prime}$. This trend is a consequence of divergence in relaxation times, which allows us to estimate a much lower freezing temperature $\left(T_{f}^{\prime} \sim 70 \mathrm{~K}\right)$. Such a quite large discrepancy between $T_{f}^{\prime}$ and $T_{f}$ must be partially ascribed to rough estimation of freezing temperature given by the VF fit, while the true value is likely affected by several factors, including compositional inhomogeneities at the local scale that may leave active relaxation processes at lower temperatures [24]. For the next discussion, we individuate an intermediate temperature $T^{\prime} \sim 100 \mathrm{~K}$ between $T_{f}^{\prime}$ and $T_{f}$, which corresponds nearly to the maximum of $\epsilon^{\prime \prime}$ and was found to be at a critical temperature in the analyses described below. "

\section{B. Polarization analysis}

Temperature-dependent P-E hysteresis loops (Fig. 2) were taken within the temperature range $80-350 \mathrm{~K}$. One can see that the zero-field polarization (remanence) is smaller in the entire temperature range. This could be due to the fact that the nanodomains are being randomly distributed and one may get a large polarization with the application of sufficiently large external electric fields, but once the field is removed the polarization value becomes small due to the randomness of the domains [25]. The remnant polarization vs temperature 

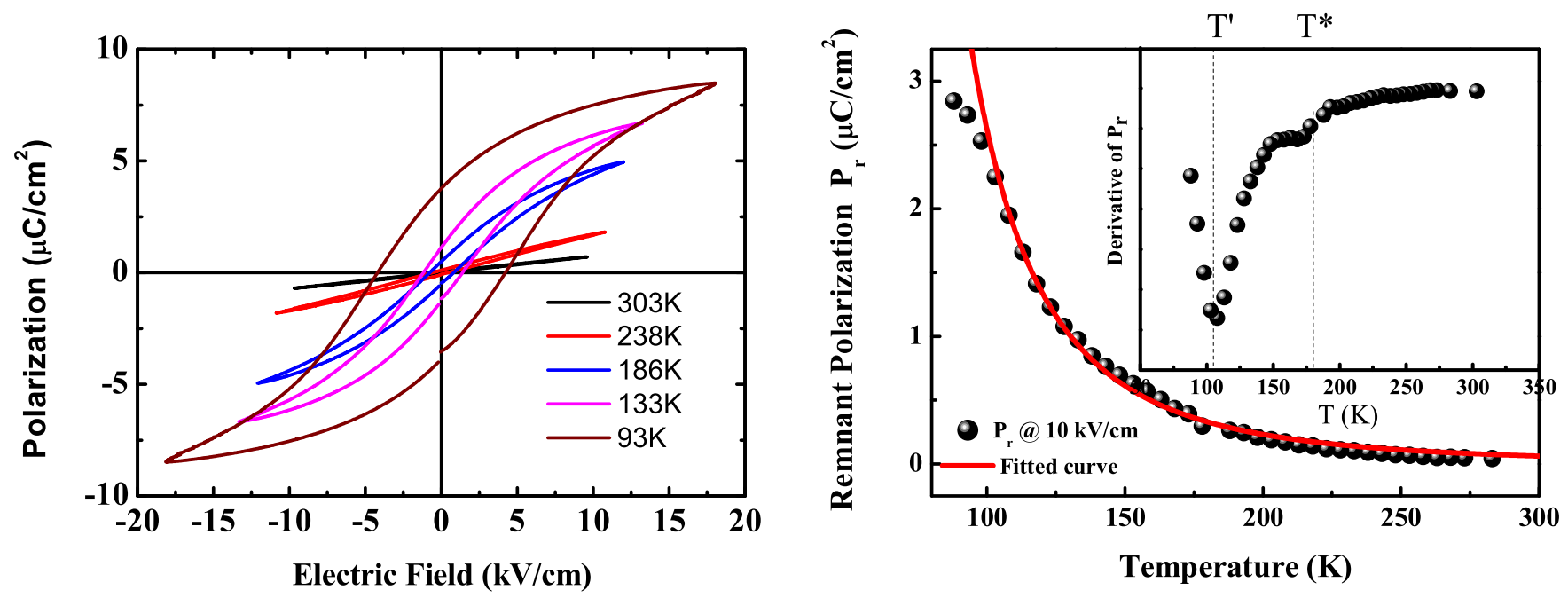

FIG. 2. Left: Ferroelectric loops (P-E) of $\mathrm{BaTi}_{0.7} \mathrm{Sn}_{0.3} \mathrm{O}_{3}$ at the indicated temperatures. Right: Remnant polarization variation with temperature with its derivative shown in the inset.

taken at high field $(10 \mathrm{kV} / \mathrm{cm})$ on warming cycle is also plotted in Fig. 2, with its derivative in the inset. The remnant polarization is found to slowly decrease on increasing temperature up to an inflection point around $T^{\prime} \sim 100 \mathrm{~K}$, signaled by a dip in derivative, then decreases smoothly until room temperature. It can be seen from the polarization derivative (inset in Fig. 2) that between $T^{\prime}$ and $T^{*}$, the polarization changes rapidly as a function of temperature. The trend of the remnant polarization as a function of temperature is fitted with a power law $\left(T-T_{a}\right)^{\alpha}$ as shown in Fig. 2. The exponent $\alpha$ is found $\sim-3$ accordingly to recent model about the growth of PNR size [26]. To understand if there are any long-range (crystallographic) and/or local structural changes associated in the region between $T^{*}$ and $T^{\prime}$ where anomalies in dielectric and polarization are seen, XRD, Raman, and XAS studies were carried out and the results are discussed in the following sections.

\section{X-ray diffraction analysis}

XRD measurements in the $50-300 \mathrm{~K}$ temperature range are carried out to study the evolution of crystallographic structure as a function of temperature. The full profile fitting analysis of XRD patterns has been carried out using the FULLPROF program [27] and the refinement reveals a cubic structure (space group 221) in the whole temperature range, a characteristic of canonical relaxors [28]. The evolution of unit-cell volume with temperature is shown in Fig. 3 and a negative thermal expansion (NTE) of $\frac{\delta V}{V}=7.5 \times 10^{-4}$ is evident in the temperature region below $T^{*}$. This trend is almost similar to the behavior observed in $\mathrm{Pb}$-based relaxors in which zero thermal expansion or weak NTE are generally reported [15].

This anomalous changes in volume may be attributed to the development of local elastic strains in the system, realized from acoustic and piezo force microscopy measurements in lead-based relaxors $[15,29]$ in the region above dielectric maxima. The XRD pattern refinement provides additional details looking at the broadening of the diffraction peaks, which are related to the coherence length of crystallites and/or larger stress strain in the sample. It is evident from the data in Fig. 3 that the full width at half maximum (FWHM) of different reflections raises on cooling below $T^{*}$ and reaches a maximum between $T^{*}$ and $T^{\prime}$, signaling some increasing structural disorder on cooling below $T^{*}$. It is noteworthy that the observed trend in FWHM is similar to that found in $\mathrm{Ba}(\mathrm{Zr}, \mathrm{Ti}) \mathrm{O}_{3}$ systems [30]. Our polarization studies show an increase in remnant polarization below $T^{*}$ (Fig. 2). This anomalous change in volume associated with onset of polarization is reminiscent of typical FE materials such as BTO, $\mathrm{PbTiO}_{3}$ etc., in the region of transition from para to FE states [31]. To achieve deeper insight about the origin of these structural changes, we performed further Raman and XAS measurements.
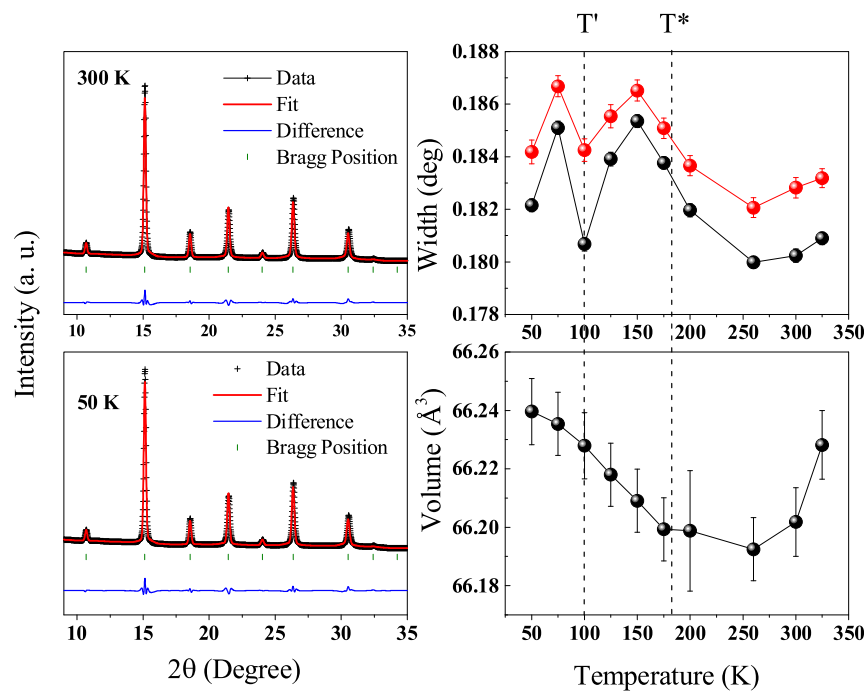

FIG. 3. Left: Representative temperature-dependent x-ray diffraction patterns of $\mathrm{BaTi}_{0.7} \mathrm{Sn}_{0.3} \mathrm{O}_{3}$ measured with $\lambda=0.7530$ $\mathrm{nm}$ radiation. The experimental data is profile fitted with FULLPROF program. Right: The variation of unit-cell volume and the full width at half maximum (FWHM) of (111) reflection (red) and (110) reflection (black). 


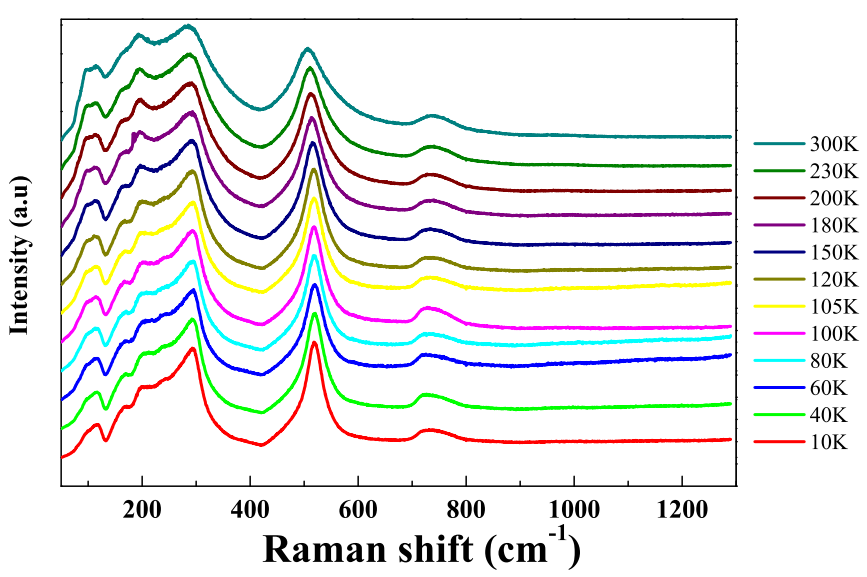

FIG. 4. Representative temperature-dependent Raman spectra of $\mathrm{BaTi}_{0.7} \mathrm{Sn}_{0.3} \mathrm{O}_{3}$.

\section{Raman spectroscopy investigation}

The Raman spectra as a function of temperature have been measured on warming cycle in the 10-300 K temperature range; representative spectra are summarized in Fig. 4. The Sn doping gives rise to some compositional disorder broadening in the Raman modes due to the mixing effect; this makes it difficult to unequivocally assign the observed features. However, the $10 \mathrm{~K}$ spectrum (Fig. 4) closely resembles the Raman modes of rhombohedral undoped BTO [32-34]. Raising the temperatures modifies the Raman spectra; to highlight the temperature effect, the raw spectra were treated [35] subtracting a linear baseline in the $120-680 \mathrm{~cm}^{-1}$ Raman shift region and normalized to the integrated intensity. Normalized spectra are shown in Fig. 5(a), the effect of temperature is evident at the positions labeled A-D; in particular, mode A around $200 \mathrm{~cm}^{-1}$ raises while the B-D peaks progressively decrease, broaden, and shift as the temperature increases. The superimposition of broad bands makes it difficult to reliably analyze the Raman spectra as a sum of independent modes. Therefore, to simplify the interpretation of the spectral features, we exploited the principal component analysis (PCA) technique using the ORANGE software [35].

The PCA is a tool of multivariate analysis used to reduce the number of variables in a multidimensional data set, retaining the variation within the data set [36]. The principal components (PCs), are the eigenvectors of the correlation matrix (Raman shift versus temperature) so each Raman spectrum as a function of temperature $R(T)$ can be reproduced as $R(T)=$ $R_{o}+\sum_{i} \alpha_{i}(T) \mathrm{PC}_{i}$ with $R_{o}$ being the average Raman spectrum over the whole temperature range, and $\alpha_{i}(T)$ the temperature dependent weight of the $i^{\text {th }}$ component. The $\mathrm{PC}_{i}$ are sorted according to the percentage of variance attributed to each in the data set, in our case the first two components shown in Fig. 5(a) accounts for the 97 at. \% of the total variance, $\sim 93$ at. $\%$ for $\mathrm{PC}_{1}$, and $\sim 4$ at. $\%$ for $\mathrm{PC}_{2}$, respectively.

The evolution of $\alpha_{i}(T)$ as a function of temperature [Fig. 5(b)] allows us to individuate spectral regions that evolve together with temperature and understand the main changes in the spectral features. In our data, $\alpha_{1}$ raises linearly on cooling with an evident change of slope around $100 \mathrm{~K}$ while $\alpha_{2}$ depicts an evident change of slope around $180 \mathrm{~K}$; these
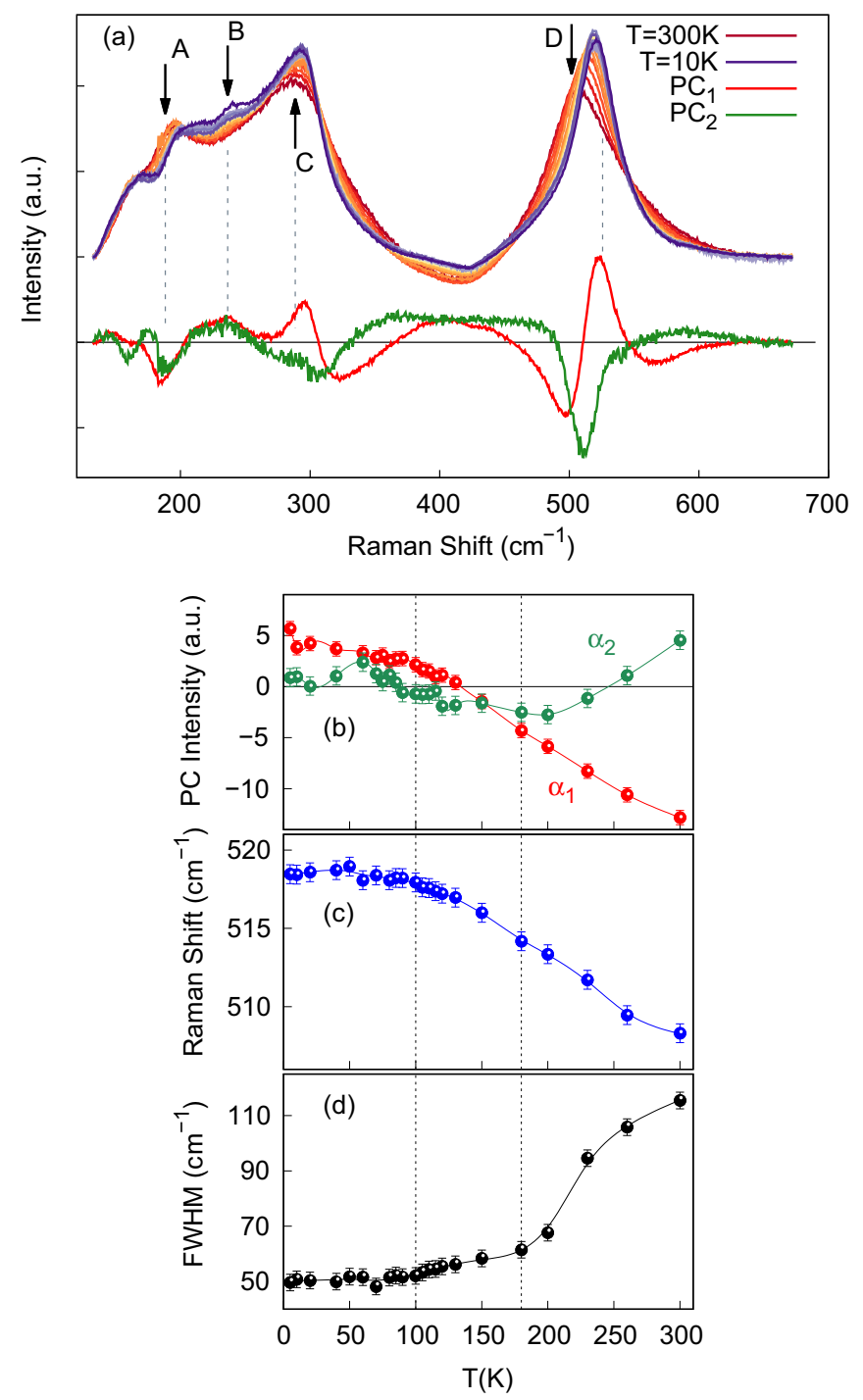

FIG. 5. Top: Normalized temperature-dependent Raman spectra of $\mathrm{BaTi}_{0.7} \mathrm{Sn}_{0.3} \mathrm{O}_{3}$ from $10 \mathrm{~K}$ to $300 \mathrm{~K}$. The first $\left(\mathrm{PC}_{1}\right)$ and second $\left(\mathrm{PC}_{2}\right)$ principal components are shown, the main changes in the Raman spectra as a function of temperature are highlighted (labels $\mathrm{A}-\mathrm{D})$. The bottom plots report the temperature evolution of the $\mathrm{PC}_{1}$, $\mathrm{PC}_{2}$ (a)Raman shift (b), and FWHM (c) for the isolated peak D. The vertical lines in the bottom frames point out the $T^{\prime}$ and $T^{*}$ temperatures.

features roughly correspond to the $T^{\prime}$ and $T^{*}$ temperatures, clearly individuating a correlation between the temperature behavior of the phonon modes and the dielectric response of the sample (Fig. 1). It must be stressed that the PCA analysis clearly shows that the evolution of the whole phonon spectrum undergoes changes as a function of temperature around $T^{\prime}$ and $T^{*}$. This trend appears to be related to the structural anomalies highlighted by XRD data.

The main Raman TO mode [peak D, Fig. 5(a)] around $510 \mathrm{~cm}^{-1}$ is well isolated and suitable for a peak fitting analysis [34], its Raman shift position, and FWHM as a function of temperature are reported in Figs. 5(c) and 5(d). The overall trend of peak position and FWHM as a function of temperature are consistent with literature results [22]. The 

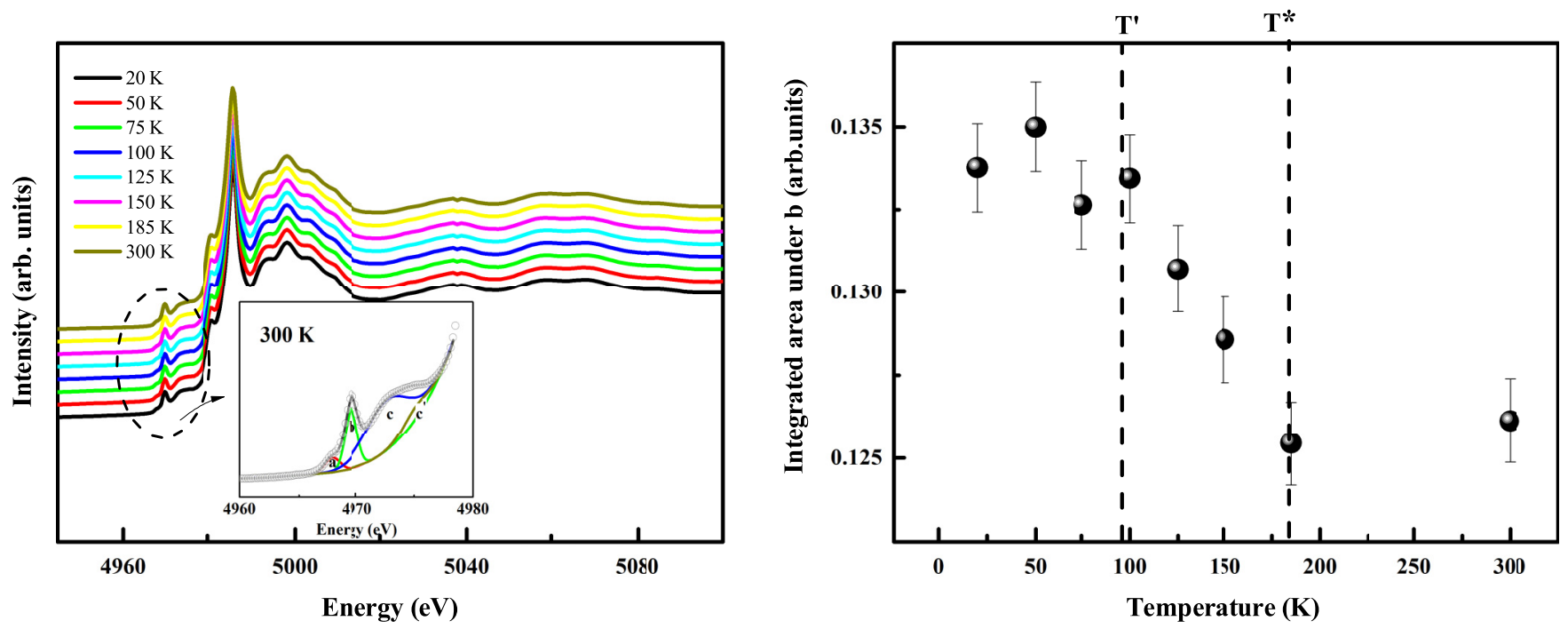

FIG. 6. Representative temperature-dependent X-ray absorption spectra (XAS) of $\mathrm{BaTi}_{0.7} \mathrm{Sn}_{0.3} \mathrm{O}_{3}$. The data is vertically shifted. Inset shows the representative fitting of different modes in pre-edge region for $300 \mathrm{~K}$ data. Right: Variation of integral area under peak b with temperature.

peak position and FWHM as a function of temperature depict an evident change of slope raising the temperature above $100 \mathrm{~K}$, while the FWHM presents a second critical change above $180 \mathrm{~K}$, these temperatures being related to the $T^{\prime}$ ad $T^{*}$ temperatures in agreement with the overall evolution of $\mathrm{PC}_{i}$ spectral weights. Summarizing the evolution of the Raman spectra as a function of temperature points out an evident relationship between phonon dynamics, dielectric properties, and crystallographic anomalies, coherently pointing out peculiar changes crossing the $T^{*}$ and $T^{\prime}$ temperatures. The Ti and $\mathrm{Sn}$ $K$-edge XAFS data have been used to achieve further details about the associated local structural changes.

\section{E. X-ray absorption spectroscopy investigation, Ti $K$ and $S n K$ edges}

Figure 6 shows the x-ray absorption spectra (XAS) measured at different temperatures across Ti $K$ edge. The closeness of $\mathrm{Ba} L_{3}$ edge $(5247 \mathrm{eV})$ with Ti $K$ edge $(4996 \mathrm{eV})$ prevents the analysis of the extended region of the XAS (EXAFS) spectra and hence the analysis is focused only on the near-edge region (XANES) of the spectra, as the pre-edge features can give relevant information on local coordination in terms of local coordination symmetry. In particular, the Ti $K$ edge pre-edge XANES features have been demonstrated to be very sensitive to the local distortions of the $\mathrm{TiO}_{6}$ octahedra. In particular, the analysis of the Ti $K$ pre-edge XANES features as a function of temperature allows us to shed light about the evolution of $\mathrm{TiO}_{6}$ distortions across the dielectric transition. The normalized temperature-dependent XANES spectra are shown in Fig. 6; they all appear very equal in the whole temperature range, corresponding to the $\mathrm{Ti}^{4+}$ valence state coordinated to six oxygen in octahedral $\mathrm{TiO}_{6}$ coordination geometry.

Looking closer at the Ti pre-edge region present are the first peak $(b)$ and a broad step (c, $\left.c^{\prime}\right)$ (inset, Fig. 6) before the raising of the main edge, for a perfect $\mathrm{TiO}_{6}$ octahedron $\left(O_{h}\right.$ symmetry), the pre-edge peak $b$ originated from the dipole-forbidden $s-d$ photoelectron transitions. Distortions of the $\mathrm{TiO}_{6}$ octahedron causes a partial $p d$ hybridization between $\operatorname{Ti}(d)$ and $\mathrm{O}(p)$ states, giving rise to the possibility of $s-p d$ dipole-allowed transitions to empty $p$-components of hybrid $p d$ levels. It has been demonstrated that the area of peak $b$ is proportional to the off-center displacement of Ti in the $\mathrm{TiO}_{6}$ octahedron and inversely proportional to unit cell volume [37-39].

The Ti XAFS spectra have been treated subtracting a linear pre-edge background and normalized to the jump-edge discontinuity following the standard procedures for XAFS data analysis. The same parameters were used for all temperatures to avoid spurious effects. The quantitative analysis of the pre-edge peak has been performed subtracting an arc-tangent function mimicing the onset of the main edge (transitions to the continuum photo electron states), and integration under peak $b$ is performed. Our values are in accordance to the literature [40] and the integral area of peak $b$ as a function of temperature is reported in the panel of Fig. 6 (right). It is evident that the peak area raises below $180 \mathrm{~K}$, reaching a broad roughly flattening on cooling around $100 \mathrm{~K}$. The effect is small but reliable above the uncertainty, thanks to the high quality and reproducibility of the Ti XANES spectra. This trend depicts a raising of $\mathrm{TiO}_{6}$ distortions below $T^{*}$ that reach a maximum close around $T^{\prime}$. These distortions likely originate from the off-center $\mathrm{Ti}$ displacement within the $\mathrm{TiO}_{6}$ octahedra as suggested by the Raman and polarization data, raising the electric dipole moment.

The Sn $K$-edge absorption data were treated using the ESTRA program [41] following the standard procedures [42] for data normalization and extraction of structural EXAFS signal $\chi(k)$. The same extraction/normalization parameters were used for all temperatures to minimize the artifacts. In Fig. 7, the Sn $K$-edge EXAFS signals $\left(k^{2}\right.$ weighted) and the moduli of their Fourier transforms $(|F T|)$ are shown. The effect of temperature is evident: The amplitude of the structural oscillations in the $k^{2} \chi(k)$ signal decreases with increasing temperature, signaling the raising of thermal 

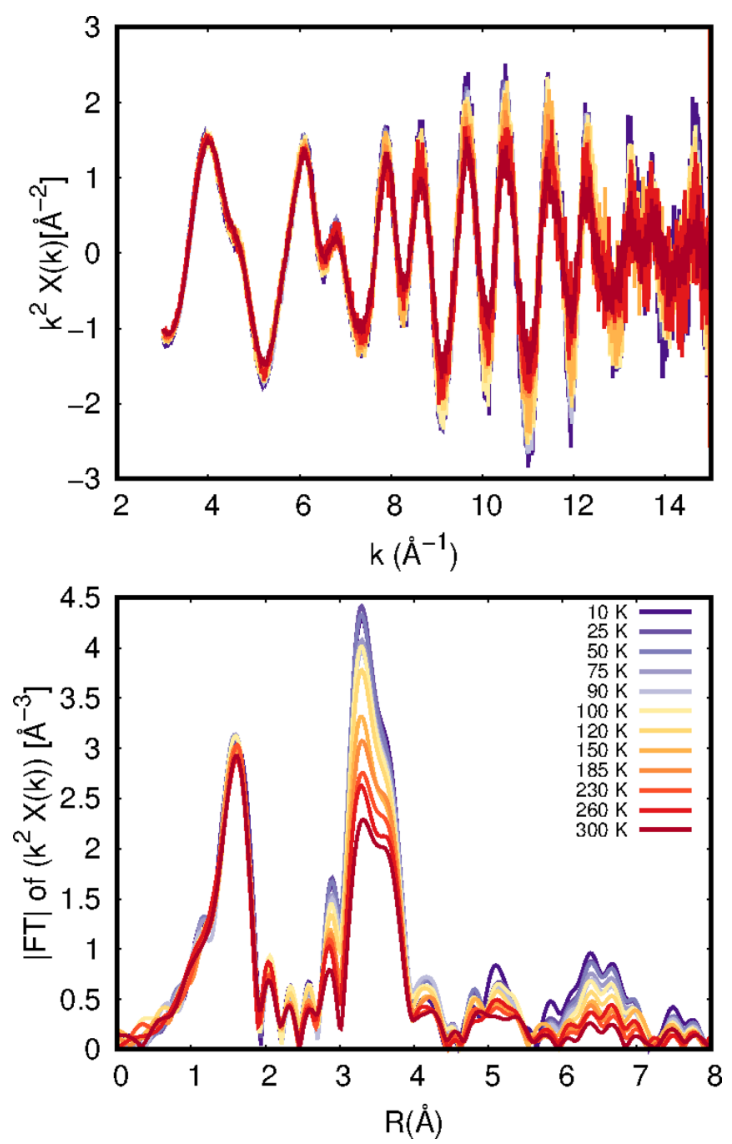

FIG. 7. Sn $K$-edge EXAFS signals $\left[k^{2} \chi(k)\right]$ and the moduli of their Fourier transforms $(|F T|)$. The amplitude of the EXAFS oscillations decrease with raising temperature, causing attenuation of the peaks in the Fourier transform.

disorder; consequently, an attenuation of the peaks in the $|F T|$ can be observed. The quantitative analysis of the Sn $K$-edge EXAFS spectra allows us to individuate the role of Sn across the dielectric transition. The quantitative analysis has been carried out fitting the raw $k^{2} \chi(k)$ spectra in $k$-space [41] in the $k$-range $3-15 \AA^{-1}$ to the model function, which is a sum of partial contribution (coordination shells). Each contribution, calculated using the standard EXAFS formula [42], assuming a Gaussian neighbor distribution, is characterized by an average coordination distance $\left(R_{i}\right)$, multiplicity number $\left(N_{i}\right)$, and mean-square relative displacement (MSRD) factor $\sigma_{i}^{2}$. The neighbor shells were individuated from the $\mathrm{BaSn}_{0.3} \mathrm{Ti}_{0.7} \mathrm{O}_{3}$ crystallographic structure, being a perovskite cubic unit cell with $\mathrm{Ti} / \mathrm{Sn}$ ions close to the cube center linked to six oxygen ions near to the cube face centres, the $\mathrm{Ba}$ ions located at the eight cube corners. Eight contributions were considered for the analysis, choosing the most relevant single (SS) and multiple (MS) scattering contributions on the basis of their amplitude and statistical significance in the fitting.

The first shell consists of the six oxygen $\left(\mathrm{O}_{1}\right)$ nearest neighbors located at about $2 \AA$, arranged in an octahedral configuration (SS contribution), for the second shell there are $8 \mathrm{Ba}\left(\mathrm{Ba}_{2}\right)(\mathrm{SS})$ at about $3.5 \AA$ and, finally, the third SS shell at around $4 \AA$, originating from $\mathrm{Ti}\left(\mathrm{Ti}_{3}\right)$ and $\mathrm{Sn}\left(\mathrm{Sn}_{3}\right)$ next neighbors, linked to the $\mathrm{Sn}$ absorber through an intermediate $\mathrm{O}_{1}$.
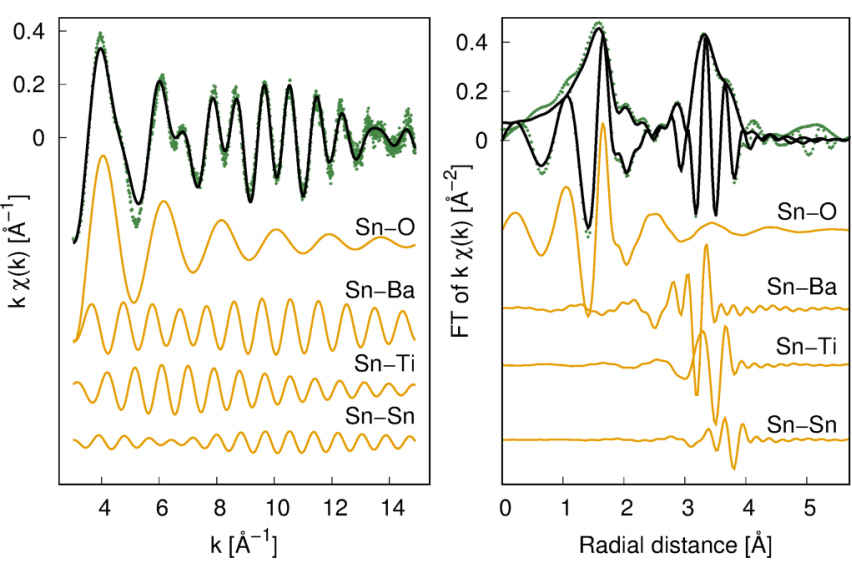

FIG. 8. Analysis of Sn $K$-edge EXAFS data. Left: Data (green dots) and best fit line (black line) in $k$-space. Right: Modulus and imaginary part of the Fourier transform of the experimental data (green dots) and best fit line (black line). Below, shifted for clarity, are reported the partial contributions used in the analysis grouped according to the various shells. The refinement demonstrates a good agreement till around $\sim 4.5 \AA$.

Due to the collinear $\mathrm{Sn}_{o}-\mathrm{O}_{1}-\mathrm{Sn}_{3} / \mathrm{Ti}_{3}$ arrangement, the multiple scattering effects are not negligible and were considered in the analysis, namely the $\mathrm{Sn}_{o}-\mathrm{O}_{1}-\mathrm{Sn}_{3} / \mathrm{Ti}_{3}-\mathrm{Sn}_{o}$ (three legs, single forward scattering) and the $\mathrm{Sn}_{o}-\mathrm{O}_{1}-\mathrm{Sn}_{3} / \mathrm{Ti}_{3}-\mathrm{O}_{1}-\mathrm{Sn}_{o}$ (four legs, double forward scattering) were considered. In principle, each coordination shell may have three structural parameters free to be refined but the correlation among them may mask their physical meaning. Therefore, preliminary tests were carried out to individuate opportune constraints among the parameters to select an optimal significant subset. The multiplicity numbers were fixed to the crystallographic values. Preliminary tests showed that $\mathrm{Sn}_{3}: \mathrm{Ti}_{3}$ ratio was close to the nominal composition value 30:70, pointing out an overall random distribution of $\mathrm{Ti}$ and $\mathrm{Sn}$ in the lattice. Therefore, the $\mathrm{Sn}_{3}: \mathrm{Ti}_{3}$ was fixed to $30: 70$ in the analysis to avoid the correlation with the MSRD parameters. Noticeably our findings differ from the XAFS studies done on $\mathrm{Ba}(\mathrm{Zr}, \mathrm{Ti}) \mathrm{O}_{3}$-based relaxors in which $\mathrm{Zr}$ segregation effect has been reported [43]. We found that the same coordination distance and the same MSRD can be used for the SS and MS of each $\mathrm{Sn}_{-} \mathrm{Ti}_{3}$ and $\mathrm{Sn}$ $\mathrm{Sn}_{3}$ configuration. Finally, the same energy shift $\Delta E$ was used for all the spectra. The free parameters used in the analysis were $\mathrm{R}_{\mathrm{SnO}}$ and $\sigma_{\mathrm{SnO}}^{2}$ for the nearest neighbor, $\mathrm{R}_{\mathrm{SnBa}}$ and $\sigma_{\mathrm{SnBa}}^{2}$, $\mathrm{R}_{\mathrm{SnSn}}$ and $\sigma_{\mathrm{SnSn}}^{2}$, and $\mathrm{R}_{\mathrm{SnTi}}$ and $\sigma_{\mathrm{SnTi}}^{2}$. This procedure allowed us to achieve a good best fit agreement in the $3-15 \AA^{-1}$ and reproduces the main structural features up to $4.5 \AA$ as shown in Fig. 8. The imposed constraints attenuate the correlations among the fitting parameters and make very reliable the weak structural modifications related to the temperature change. The coordination distances $\mathrm{R}_{i}$ are reported in Fig. 9; they are in agreement with the crystallographic structure with only weak changes as a function of temperature, noticeably the average $\mathrm{R}_{\mathrm{SnTi}}=4.02 \AA$ distances are shorter than $\mathrm{R}_{\mathrm{SnSn}}=4.08$ $\AA$, accordingly, to the smaller ionic radius of $\mathrm{Ti}(0.61 \AA)$ respect to $\mathrm{Sn}(0.71 \AA)$.

The MSRDs increase raising the temperature (Fig. 10), pointing out the increasing thermal disorder $[44,45]$. The 


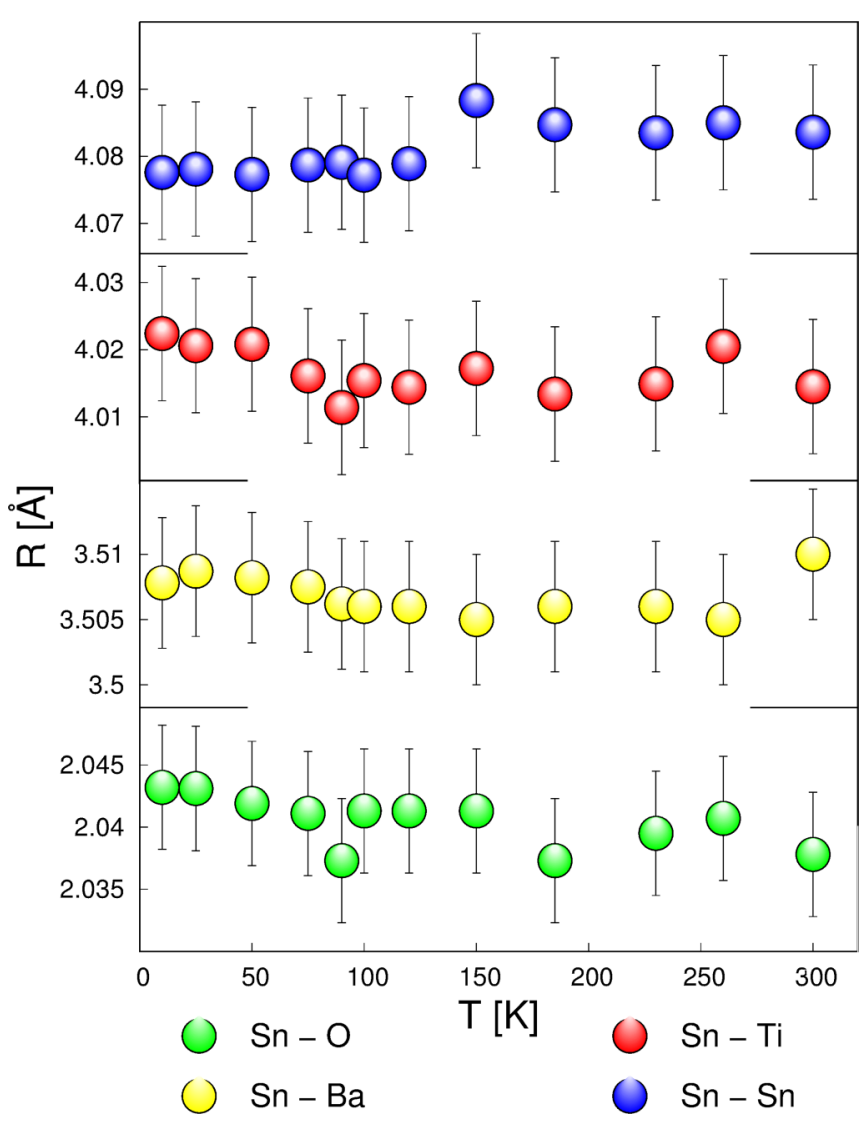

FIG. 9. Coordination distances as a function of temperature for all the shells involved in the analysis. They are in agreement with the crystallographic structure with only weak changes as a function of temperature. The average $\mathrm{R}_{\mathrm{SnTi}}$ distances are shorter than $\mathrm{R}_{\mathrm{SnSn}}$, accordingly, to the smaller ionic radius of Ti respect to $\mathrm{Sn}$.

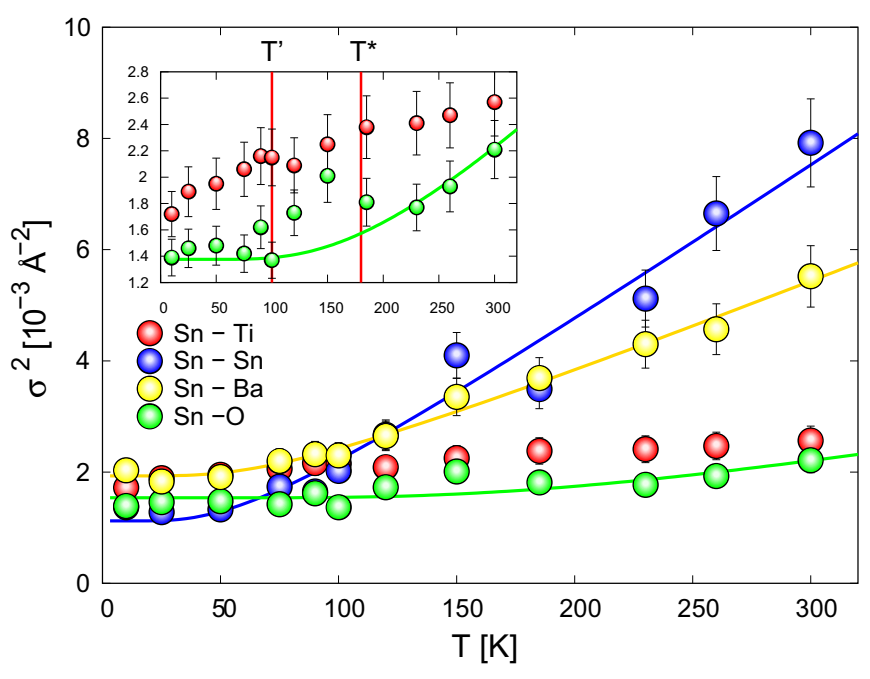

FIG. 10. Temperature dependence of mean square relative displacement (MSRD) $\left[\sigma^{2}\right]$ parameters for all shells involved in the analysis. The insert shows a change at roughly $120-180 \mathrm{~K}$ in the $\sigma_{S n-O}^{2}$ behavior.
MSRD data were fitted with a constant term $\sigma_{s}^{2}$, representing the static contribution to the disorder, plus a temperaturedependent term we modeled using a correlated Einstein model [46]:

$$
\sigma^{2}=\sigma_{s}^{2}+\frac{\hbar}{2 \mu \omega_{E}} \operatorname{coth}\left(\frac{\hbar \omega_{E}}{2 k_{B} T}\right) .
$$

Here $\mu$ is the reduced mass of the pairs and $\omega_{E}$ is the Einstein frequency related to the Einstein temperature as $\theta_{E}=$ $\hbar \omega_{E} / k_{B}$. The $\theta_{E}$ of Sn-O $\mathrm{O}_{1}(\sim 600 \mathrm{~K}), \mathrm{Sn}-\mathrm{Ba}_{2}(\sim 280 \mathrm{~K})$, and $\mathrm{Sn}_{-} \mathrm{Sn}_{3}(\sim 169 \mathrm{~K})$ progressively decrease for the more and more distant shells, signaling the phonon activation energy reduction for the furthest away shells. Interestingly, the $\sigma^{2}(T)$ of $\mathrm{Sn}-\mathrm{O}_{1}$ and $\mathrm{Sn}_{-} \mathrm{Ti}_{3}$ shells clearly depart from the Einstein model behavior, suggesting anomalies in the evolution of thermally activated disorder. In particular, the $\sigma^{2}(T)$ of Sn-O shell departs from the Einstein by more than 30 at. \%, well above the statistical uncertainty, exactly in the $T^{\prime}-T^{*}$ interval, being the temperature region in which anomalies have already been revealed coherently by XRD, Raman, and Ti- $K$-edge XANES, therefore supporting that these effects are real and not artifacts of the analysis. It must also be noticed that comparing the MSRD of $\mathrm{Sn}-\mathrm{Sn}_{3}$ and $\mathrm{Sn}-\mathrm{Ti}_{3}$ shells, the last one grows much slower as a function of temperature in the $1.7 \times 10^{-3}-2.6 \times 10^{-3} \AA^{2}$ range with a roughly linear trend. Such a behavior could be ascribed to correlations between Sn-O and O-Ti bond disorder along the Sn-O-Ti chains that would raise the phonon activation energy. Such a correlation between $\mathrm{Sn}-\mathrm{O}$ and Ti-O bonds is likely related to the anomaly observed on the $\sigma^{2}(T)$ of the Sn-O shell, pointing out an indirect involvement of $\mathrm{Sn}$ local structure in the polarization. Noticeably, the $\mathrm{Zr} K$-edge EXAFS studies on $\mathrm{BaTi}_{1-\mathrm{x}} \mathrm{Zr}_{\mathrm{x}} \mathrm{O}_{3}$ relaxors [43] revealed larger static disorder around $\mathrm{Zr}$ in the presence of $\mathrm{Ti}(\mathrm{x} \sim 0.3$ ), pointing out also in this case that the presence of $\mathrm{Ti}$ altered the $\mathrm{Sn}-\mathrm{O}$ bond length distribution. Discovering that the local structure around $\mathrm{Sn}$ is involved, albeit indirectly, in the dielectric response of $\mathrm{BaTi}_{1-\mathrm{x}} \mathrm{Sn}_{\mathrm{x}} \mathrm{O}_{3}$ is a relevant finding that we corroborated with temperature dependent ${ }^{119} \mathrm{Sn}$ Mössbauer measurements on the same sample as discussed below.

\section{F. Mössbauer analysis}

In displacive like FE transitions, loss of inversion symmetry is associated with an onset of polarization may result in changes in interaction between nuclear quadrapole moment and electric field gradient (EFG). It is to be noted that a direct relation between polarization, local distortion, and EFG is reported in FE materials [47]. Various experimental reports have also been published, indicating the relation between FE polarization and EFG [47]. ${ }^{119} \mathrm{Sn}$ and ${ }^{57} \mathrm{Fe}$ Mössbauer measurements and NMR measurements are reported in materials such as $\mathrm{BaTiO}_{3}, \mathrm{PbZrO}_{3}$ etc., to study the FE transitions by tracking the temperature variation of various hyperfine parameters [48-51]. Temperaturedependent ${ }^{119} \mathrm{Sn}$ Mössbauer measurements are carried out in the present paper to study the changes in hyperfine parameters with temperature and the data is shown in Fig. 11. Almost zero center shift indicates a $\mathrm{Sn}^{4+}$ oxidation state throughout the temperature range. The evolution in the width of the 

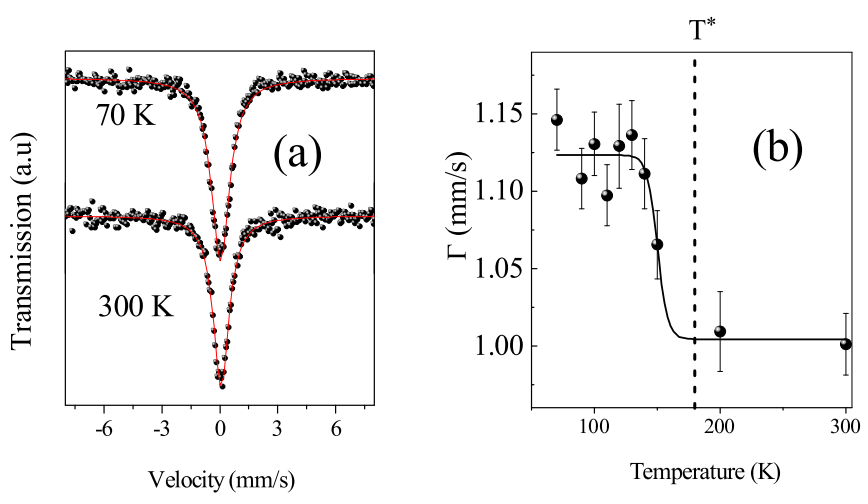

FIG. 11. Left: Representative temperature-dependent ${ }^{119} \mathrm{Sn}$ Mössbauer spectra of $\mathrm{BaTi}_{0.7} \mathrm{Sn}_{0.3} \mathrm{O}_{3}$. Right: Temperature variation of width of ${ }^{119} \mathrm{Sn}$ Mössbauer spectra, considered to be proportional to the electric field gradient.

Mössbauer spectra $\Gamma$, which is considered to be directly proportional to the strength of EFG around $\mathrm{Sn}$ nucleus $[2,48-50]$, is reported as a function of the temperature in Fig. 11(b). The $\Gamma$ increases significantly on cooling below $200 \mathrm{~K}$ and stabilizes below $150 \mathrm{~K}$ and a similar trend in EFG is also seen in compounds that exhibit FE phase transitions [2] and lower Sn content. This finding definitively corroborates a relationship between local structural changes around $\mathrm{Sn}$ related to the $\mathrm{TiO}_{6}$ polarization deformation in this system.

\section{SUMMARY AND CONCLUSIONS}

We have demonstrated the relaxor behavior in the $30 \% \mathrm{Sn}-$ doped $\mathrm{BaTiO}_{3}$ system from dielectric and polarization data, revealing dispersion in dielectric constant, slim hysteresis loops, and remnant polarization well above the dielectric temperature maximum $\left(T_{m}\right)$. Combining XRD, Raman, XAFS, and Mössbauer spectroscopy, we were able to coherently identify local and crystallographic structural counterparts associated with the relaxor properties. The real part of dielectric permittivity develops a frequency dispersion below $T^{*} \sim 180 \mathrm{~K}$ while the imaginary part of permittivity, signaling dielectric losses, increases reaching the maximum around $T^{\prime} \sim 100 \mathrm{~K}$. $\mathrm{XRD}$ results demonstrate crystallographic distortions active below $T^{*}$ being associated with NTE. The evolution of Raman spectra as a function of temperature depict changes associated to $T^{*}$ and $T^{\prime}$, showing a broad involvement of the entire phonon spectrum in changes associated with the macroscopic relaxor behavior. The Ti $K$-edge XANES analysis confirms the enhancement of the off-center displacement of $\mathrm{Ti}$ in $\mathrm{TiO}_{6}$ octahedra below $T^{*}$. The analysis of Sn $K$-edge EXAFS spectra originally demonstrated Sn local structure modifications directly involved in structural changes related to the evolution of macroscopic relaxor properties, suggesting a role of the vibrational modes associated with Sn sublattices within the PNR dynamics. This finding has been further corroborated by ${ }^{119} \mathrm{Sn}$ Mössbauer results, suggesting changes in EFG around the Sn nucleus at the onset of polarization below $T^{*}$. Combining complementary probes, we have been able to relate the relaxor properties of $\mathrm{BaSn}_{0.3} \mathrm{Ti}_{0.7} \mathrm{O}_{3}$ to the structural changes at the atomic scale, hence drawing a connection with the dipole dynamics and providing a deeper understanding beyond the phenomenological models $[10,16]$. Our experimental results provide evidence which may validate some of the recent theoretical works done on lead-free $\mathrm{Ba}(\mathrm{Zr}, \mathrm{Ti}) \mathrm{O}_{3}$, where it has been suggested that the temperature evolution of the coupling parameter between the $\mathrm{Zr}$ and $\mathrm{Ti}$ sites $[7,8,20]$ is responsible for relaxor behavior.

\section{ACKNOWLEDGMENTS}

A.S. and V.R.R. thank the Department of Science and Technology (DST) and IISc, Bangalore for travel support for performing experiments at XAFS beamline, ELETTRA No. INT/ITALY/P-22/2016 (SP). V.R.R. would like to acknowledge financial support by the Department of Science and Technology (Government of India) provided within the framework of the India@DESY collaboration for measurements at PETRA-III. Ms. Anjali, Ms. Zainab and Mr. Manik are thanked for the help during XAFS measurements. Thanks are due to Dr. Luca Olivi for help during XAFS measurements at Elettra. Mr. Anil Gome is acknowledged for help in Mössbauer and Mr. M. N. Singh is acknowledged for help in LT XRD measurements. V.R.R. thanks Prof. Ajay Gupta for discussions and encouragement.
[1] S.-E. Park and T. R. Shrout, Ultrahigh strain and piezoelectric behavior in relaxor based ferroelectric single crystals, J. Appl. Phys. 82, 1804 (1997).

[2] S. K. Upadhyay, V. R. Reddy, P. Bag, R. Rawat, S. Gupta, and A. Gupta, Electro-caloric effect in lead-free Sn-doped $\mathrm{BaTiO}_{3}$ ceramics at room temperature and low applied fields, Appl. Phys. Lett. 105, 112907 (2014).

[3] G. Burns and F. Dacol, Crystalline ferroelectrics with glassy polarization behavior, Phys. Rev. B 28, 2527 (1983).

[4] L. Xie, Y. Li, R. Yu, Z. Cheng, X. Wei, X. Yao, C. Jia, K. Urban, A. Bokov, Z.-G. Ye et al., Static and dynamic polar nanoregions in relaxor ferroelectric $\mathrm{Ba}\left(\mathrm{Ti}_{1-x} \mathrm{Sn}_{x}\right) \mathrm{O}_{3}$ system at high temperature, Phys. Rev. B 85, 014118 (2012).

[5] C. A. Randall and A. Bhalla, Nanostructural-property relations in complex lead perovskites, Jpn. J. Appl. Phys. 29, 327 (1990).
[6] M. E. Manley, J. W. Lynn, D. Abernathy, E. Specht, O. Delaire, A. Bishop, R. Sahul, and J. Budai, Phonon localization drives polar nanoregions in a relaxor ferroelectric, Nat. Commun. 5, 3683 (2014).

[7] C. Mentzer, S. Lisenkov, Z. Fthenakis, and I. Ponomareva, Phase evolution in the ferroelectric relaxor $\mathrm{Ba}\left(\mathrm{Ti}_{1-x}, \mathrm{Zr}_{x}\right) \mathrm{O}_{3}$ from atomistic simulations, Phys. Rev. B 99, 064111 (2019).

[8] D. Wang, J. Hlinka, A. Bokov, Z.-G. Ye, P. Ondrejkovic, J. Petzelt, and L. Bellaiche, Fano resonance and dipolar relaxation in lead-free relaxors, Nat. Commun. 5, 5100 (2014).

[9] S. Tinte, B. P. Burton, E. Cockayne, and U. Waghmare, Origin of the Relaxor State in $\mathrm{Pb}\left(\mathrm{B}_{x} \mathrm{~B}{ }_{1-x}\right) \mathrm{O}_{3}$ Perovskites, Phys. Rev. Lett. 97, 137601 (2006).

[10] V. Westphal, W. Kleemann, and M. Glinchuk, Diffuse Phase Transitions and Random-Field-Induced Domain States of the 
'Relaxor" Ferroelectric $\mathrm{PbMg}_{1 / 3} \mathrm{Nb}_{2 / 3} \mathrm{O}_{3}$, Phys. Rev. Lett. 68, 847 (1992).

[11] R. Pirc, Z. Kutnjak, and N. Novak, Compressible spherical dipolar glass model of relaxor ferroelectrics, J. Appl. Phys. 112, 114122 (2012).

[12] A. Akbarzadeh, S. Prosandeev, E. J. Walter, A. Al-Barakaty, and L. Bellaiche, Finite-Temperature Properties of $\mathrm{Ba}(\mathrm{Zr}, \mathrm{Ti}) \mathrm{O}_{3}$ Relaxors from First Principles, Phys. Rev. Lett. 108, 257601 (2012).

[13] J. Toulouse, The three characteristic temperatures of relaxor dynamics and their meaning, Ferroelectrics 369, 203 (2008).

[14] B. Hehlen, M. Al-Sabbagh, A. Al-Zein, and J. Hlinka, Relaxor Ferroelectrics: Back to the Single-Soft-Mode Picture, Phys. Rev. Lett. 117, 155501 (2016).

[15] B. Dkhil, P. Gemeiner, A. Al-Barakaty, L. Bellaiche, E. Dul'kin, E. Mojaev, and M. Roth, Intermediate temperature scale $T^{*}$ in lead-based relaxor systems, Phys. Rev. B 80, 064103 (2009).

[16] W. Kleemann, Random fields in relaxor ferroelectrics-a jubilee review, J. Adv. Dielect. 2, 1241001 (2012).

[17] Y. Moriya, H. Kawaji, T. Tojo, and T. Atake, Specific-Heat Anomaly Caused by Ferroelectric Nanoregions in $\mathrm{Pb}\left(\mathrm{Mg}_{1 / 3}\right.$ $\left.\mathrm{Nb}_{2 / 3}\right) \mathrm{O}_{3}$ and $\mathrm{Pb}\left(\mathrm{Mg}_{1 / 3} \mathrm{Ta}_{2 / 3}\right) \mathrm{O}_{3}$ Relaxors, Phys. Rev. Lett. 90, 205901 (2003).

[18] A. Levstik, Z. Kutnjak, C. Filipič, and R. Pirc, Glassy freezing in relaxor ferroelectric lead magnesium niobate, Phys. Rev. B 57, 11204 (1998).

[19] M. Nagasawa, H. Kawaji, T. Tojo, and T. Atake, Absence of the heat capacity anomaly in the $\mathrm{Pb}$-free relaxor $\mathrm{BaTi}_{0.65} \mathrm{Zr}_{0.35} \mathrm{O}_{3}$, Phys. Rev. B 74, 132101 (2006).

[20] I. Grinberg, P. Juhás, P. K. Davies, and A. M. Rappe, Relationship between Local Structure and Relaxor Behavior in Perovskite Oxides, Phys. Rev. Lett. 99, 267603 (2007)

[21] A. Di Cicco, G. Aquilanti, M. Minicucci, E. Principi, N. Novello, A. Cognigni, and L. Olivi, Novel XAFS capabilities at ELETTRA synchrotron light source, J. Phys.: Conf. Ser. 190, 012043 (2009).

[22] A. Kumar, I. Rivera, and R. Katiyar, Investigation of local structure of lead-free relaxor $\mathrm{Ba}\left(\mathrm{Ti}_{0.70} \quad \mathrm{Sn}_{0.30}\right) \mathrm{O}_{3}$ by raman spectroscopy, J. Raman Spectrosc. 40, 459 (2009).

[23] C. Lei, A. A. Bokov, and Z.-G. Ye, Ferroelectric to relaxor crossover and dielectric phase diagram in the $\mathrm{BaTiO}_{3}-\mathrm{BaSnO}_{3}$ system, J. Appl. Phys. 101, 084105 (2007).

[24] W. Kleemann, S. Miga, J. Dec, and J. Zhai, Crossover from ferroelectric to relaxor and cluster glass in $\mathrm{BaTi}_{1-x} \mathrm{Zr}_{x} \mathrm{O}_{3}(x=$ $0.25-0.35)$ studied by non-linear permittivity, Appl. Phys. Lett. 102, 232907 (2013).

[25] L. E. Cross, Relaxor ferroelectrics, Ferroelectrics 76, 241 (1987).

[26] V. Polinger and I. B. Bersuker, Origin of polar nanoregions and relaxor properties of ferroelectrics, Phys. Rev. B 98, 214102 (2018).

[27] J. Rodríguez-Carvajal, Recent advances in magnetic structure determination by neutron powder diffraction, Physica B (Amsterdam) 192, 55 (1993).

[28] N. Yasuda, H. Ohwa, and S. Asano, Dielectric properties and phase transitions of $\mathrm{Ba}\left(\mathrm{Ti}_{1-x} \mathrm{Sn}_{x}\right) \mathrm{O}_{3}$ solid solution, Jpn. J. Appl. Phys. 35, 5099 (1996).
[29] V. V. Shvartsman, J. Dec, T. Łukasiewicz, A. L. Kholkin, and W. Kleemann, Evolution of the polar structure in relaxor ferroelectrics close to the curie temperature studied by piezoresponse force microscopy, Ferroelectrics 373, 77 (2008).

[30] P. Sciau and A.-M. Castagnos, Comparison between $\mathrm{BaTi}_{0.65}$

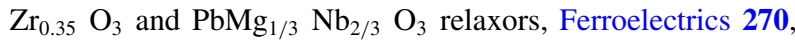
259 (2002).

[31] K. Takenaka, Progress of research in negative thermal expansion materials: Paradigm shift in the control of thermal expansion, Front. Chem. 6, 267 (2018).

[32] J. Parsons and L. Rimai, Raman spectrum of $\mathrm{BaTiO}_{3}$, Solid State Commun. 5, 423 (1967).

[33] V. Buscaglia, S. Tripathi, V. Petkov, M. Dapiaggi, M. Deluca, A. Gajović, and Y. Ren, Average and local atomic-scale structure in $\mathrm{BaZr}_{x} \mathrm{Ti}_{1-x} \mathrm{O}_{3}(x=0.10,0.20,0.40)$ ceramics by high-energy $\mathrm{X}$-ray diffraction and raman spectroscopy, J. Phys.: Condens. Matter 26, 065901 (2014).

[34] A. Dixit, S. Majumder, R. Katiyar, and A. Bhalla, Studies on the relaxor behavior of sol-gel derived $\mathrm{Ba}\left(\mathrm{Zr}_{x} \quad \mathrm{Ti}_{1-x}\right) \mathrm{O}_{3}$ $(0.30 \leqslant x \leqslant 0.70)$ thin films, in Frontiers of Ferroelectricity (Springer US, Boston, MA, 2007), pp. 87-96.

[35] J. Demšar, T. Curk, A. Erjavec, Č. Gorup, T. Hočevar, M. Milutinovič, M. Možina, M. Polajnar, M. Toplak, A. Starič et al., Orange: Data mining toolbox in Python, J. Mach. Learn. Res. 14, 2349 (2013).

[36] K. Varmuza and P. Filzmoser, Introduction to Multivariate Statistical Analysis in Chemometrics, 1st ed. (CRC Press, Boca Raton, FL, 2009).

[37] B. Ravel, E. Stern, R. Vedrinskii, and V. Kraizman, Local structure and the phase transitions of $\mathrm{BaTiO}_{3}$, Ferroelectrics 206, 407 (1998).

[38] R. Vedrinskii, V. Kraizman, A. Novakovich, P. V. Demekhin, and S. Urazhdin, Pre-edge fine structure of the $3 \mathrm{~d}$ atom K x-ray absorption spectra and quantitative atomic structure determinations for ferroelectric perovskite structure crystals, J. Phys.: Condens. Matter 10, 9561 (1998).

[39] J. Itié, B. Couzinet, A. Polian, A. Flank, and P. Lagarde, Pressure-induced disappearance of the local rhombohedral distortion in $\mathrm{BaTiO}_{3}$, Europhys. Lett. 74, 706 (2006).

[40] I. Levin, E. Cockayne, V. Krayzman, J. C. Woicik, S. Lee, and C. A. Randall, Local structure of $\mathrm{Ba}(\mathrm{Ti}, \mathrm{Zr}) \mathrm{O}_{3}$ perovskite-like solid solutions and its relation to the band-gap behavior, Phys. Rev. B 83, 094122 (2011).

[41] C. Meneghini, F. Bardelli, and S. Mobilio, ESTRA-FitEXA: A software package for EXAFS data analysis, Nucl. Instrum. Methods Phys. Res., Sect. B 285, 153 (2012).

[42] J. J. Rehr and R. C. Albers, Theoretical approaches to x-ray absorption fine structure, Rev. Mod. Phys. 72, 621 (2000).

[43] C. Laulhé, F. Hippert, J. Kreisel, M. Maglione, A. Simon, J. Hazemann, and V. Nassif, EXAFS study of lead-free relaxor ferroelectric $\mathrm{BaTi}_{1-x} \mathrm{Zr}_{x} \mathrm{O}_{3}$ at the $\mathrm{Zr} K$ edge, Phys. Rev. B 74, 014106 (2006).

[44] G. Beni and P. Platzman, Temperature and polarization dependence of extended x-ray absorption fine-structure spectra, Phys. Rev. B 14, 1514 (1976).

[45] P. Fornasini and R. Grisenti, On EXAFS debye-waller factor and recent advances, J. Synchrotron Rad. 22, 1242 (2015).

[46] G. Bunker, Introduction to XAFS: A Practical Guide to X-ray Absorption Fine Structure Spectroscopy (Cambridge University Press, Cambridge, UK, 2010). 
[47] J. Gonçalves, A. Stroppa, J. Correia, T. Butz, S. Picozzi, A. Fenta, and V. Amaral, Ab initio study of the relation between electric polarization and electric field gradients in ferroelectrics, Phys. Rev. B 86, 035145 (2012).

[48] V. Bhide and M. Multani, Mössbauer effect for Fe 57 in ferroelectric $\mathrm{BaTiO}_{3}$. II. The vacancy-impurity associated state, Phys. Rev. 149, 289 (1966).
[49] V. Bhide and V. Durge, Sn119 Mössbauer studies of lower transitions in barium titanate, Solid State Commun. 10, 401 (1972).

[50] A. Jain, S. Shringi, and M. Sharma, Temperature-dependent optical mode in antiferroelectric $\mathrm{PbZrO}_{3}$ by the Mössbauer effect, Phys. Rev. B 2, 2756 (1970).

[51] E. A. Stern, Character of Order-Disorder and Displacive Components in Barium Titanate, Phys. Rev. Lett. 93, 037601 (2004). 